\title{
Osteotomien - Becken
}

\author{
Tim Pohlemann, Jörg Holstein, Ulf Culemann
}

\section{Zusammenfassung}

Operativ korrekturbedürftige Fehlstellungen sind nach moderner, korrekt durchgeführter Primärbehandlung von Beckenringverletzungen und Azetabulumfrakturen in Deutschland selten. Unterschieden werden Pseudarthrosen und knöcherne Heilungen in Fehlstellung. Die Indikationsstellung erfolgt nach umfassender Evaluation, die neben allen Aspekten der klinischen (einschließlich neurologischer und urologischer Untersuchung) und radiologischen Untersuchung (Nativaufnahmen in verschiedenen Projektionen, CT mit Rekonstruktion und ggf. MRI-Untersuchung) ggf. auch eine psychologische Evaluation umfasst, um posttraumatische Fehlverarbeitungen auszuschließen. Die Planung ist immer individuell. Insbesondere die Frage, ob in der Sequenz Rückenlage-Bauchlage-Rückenlage oder Bauchlage-Rückenlage-Bauchlage vorgegangen wird, ist erst nach genauester Berücksichtigung aller Befunde zu entscheiden. Nach instabilen Beckenverletzungen ist aber immer eine komplette Durchtrennung des vor- deren und des hinteren Beckenrings erforderlich, mit der kompletten Lösung des Beckenbodens, einschließlich der Bandverbindungen. Diese risikoreichen Operationen erfordern eine vorbereitete, ausgezeichnete personelle, apparative und organisatorische Infrastruktur und sollten daher spezialisierten Zentren vorbehalten bleiben. Bei guter Indikationsstellung und Durchführung lässt sich allerdings die Lebensqualität der betroffenen Patienten in vielen Fällen deutlich verbessern.

\section{Osteotomies: Pelvis}

With the presently available methods of operative and non-operative fracture care, assuming state of the art surgical treatment, a late reconstruction after non-united or mal-healed pelvic ring injury is rarely necessary, also after mal-healed acetabular fractures. Before a surgical correction can be recommended to the patient, the full range of clinical (including neurological and urological examinations) and radiographic evaluations (including full sets of plain radiographs, CT examinations and in some cases MRI examinations) should be carried out and interpreted. The psychological situation of the patient has to be evaluated as well to exclude post-traumatic disorders, with a focus on the pelvic situation. The surgical planning has to be highly individual, especially the sequence of patient positioning (supine-prone-supine vs. pronesupine-prone) must be based on the individual injury pattern. However, in all cases of a translational deformity, the anterior as well as the posterior pelvic ring must be osteotomised completely, and the complete pelvic floor has to be dissected completely, including the pelvic floor ligaments. These types of operations have various risks and, besides a good preparation, an excellent infrastructure in respect of human resources, intensive care capacity, bloodbank, instruments etc. should be available. Therefore, in the German setting, a referral of such rare cases to specialised centres is recommended. After an appropriate patient selection and surgical treatment, however, the quality of life for the majority of patients can be improved significantly.

\section{Einleitung}

Beckenringverletzungen umfassen lediglich 3\% aller Frakturen und sind damit relativ selten. In den letzten Jahren wurden, nicht zuletzt durch die intensive Arbeit der Arbeitsgruppe Becken der Deutschen AO und der DGU, sehr verlässliche Behandlungskonzepte erarbeitet, die es erlauben, Notfallsituationen frühzeitig zu erkennen und auch schon in der Frühphase eine exakte Reposition und Stabilisierung z.B. mit Beckenzwinge oder Fi-

OP-JOURNAL 2011; 27: 234-238

(c) Georg Thieme Verlag KG Stuttgart · New York DOI http://dx.doi.org/10.1055/s-0031-1298187 xateur externe vorzunehmen. Auch die definitiven Behandlungsmaßnahmen mit offenen oder geschlossenen Einrichtungen, Platten- und/oder Schraubenosteosynthesen, letztere zunehmend auch perkutan und ggf. mit Navigationskontrolle, gehören in vielen größeren Krankenhäusern zum Standardrepertoire. Auch in dieser Zeitschrift wurde mehrfach über Prinzipien und Techniken berichtet. Die in Deutschland geübte Praxis, Patienten frühzeitig zu speziellen Behandlungszentren weiterzuverlegen (Stichwort: Traumanetzwerke), führt dazu, dass operativ korrekturbedürftige Fehlstellungen nach instabilen Beckenringverletzungen und auch nach Azetabulumfrakturen äußerst selten sind. Das gestiegene Verständnis der Beckenanatomie und Pathobiomechanik, die verbesserten Visualisierungsmöglichkeiten und die zunehmende operative Erfahrung am Becken versetzt uns allerdings zunehmend in die Lage, auch nach Pseudarthrosen und Heilungen in behindernden Fehlstellungen rekonstruktive Eingriffe zu empfehlen und durchzuführen $[1,3,4,6-9]$. Auch die zunehmende internationale Mobilität betroffener Patienten erhöht die Operationsfrequenz (Tab. 1). 
Tab. 1 Übersicht über Indikationen und Fälle von Spätkorrekturen an Becken und Azetabulum (Hannover und Homburg/Saar).

\begin{tabular}{lllll} 
& & $\begin{array}{l}\text { MHH } \\
\text { 1996-2000 }\end{array}$ & $\begin{array}{l}\text { Homburg } \\
\text { 2001-2011 }\end{array}$ & gesamt \\
\hline Beckenring & nach Typ C & 4 & 3 & $\mathbf{7}$ \\
\hline & nach Typ B & 1 & 4 & $\mathbf{5}$ \\
\hline & Pseudarthrose & 1 & 7 & $\mathbf{8}$ \\
\hline Beckenring bei geriatrischen Patienten & & 12 & $\mathbf{1 2}$ \\
\hline Azetabulum & 2 & 7 & $\mathbf{9}$ \\
\hline & & & & $\mathbf{4 1}$
\end{tabular}
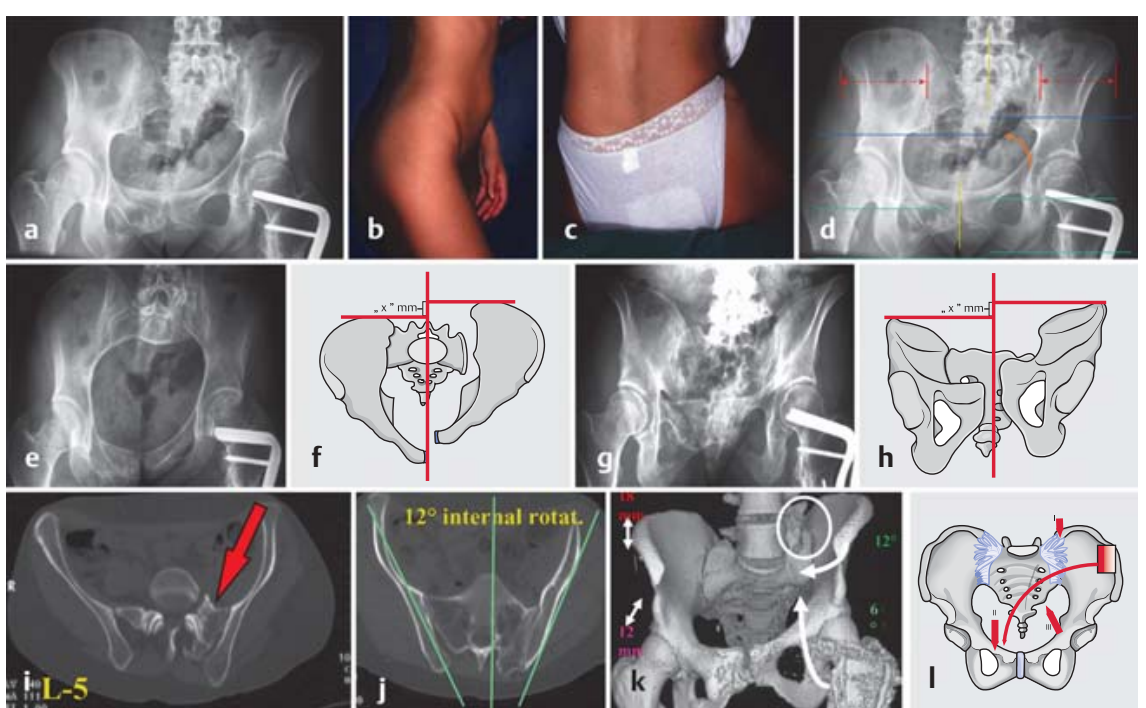

Abb. 1 a bis I a 25-jährige Patientin 1 Jahr nach Polytrauma mit fehlverheilter Beckenringverletzung vom Typ C (transpubisch rechts, transforaminal links) mit inkompletter Läsion Plexus lumbosacralis links und erheblichen Beschwerden (Fall in Teilen aus: Tscherne, Pohlemann. Unfallchirurgie Becken und Acetabulum. Heidelberg: Springer; 1998: 271). b Situation im Stand, deutliche Beinverkürzung links (mit Brettchenmethode gemessen $3 \mathrm{~cm}$ ). c Situation im Sitzen: deutliches Absinken links im Sinne der „Sitting Imbalance“. d Planung mit entsprechenden Hilfslinien anhand der a.-p. Aufnahme: linke Beckenhälfte steht kranial, dorsal und nach innen rotiert. e Inletaufnahme. $\mathbf{f}$ Messung in der Inletprojektion. g Outletaufnahme. h Messung in der Outletprojektion. i Detailanalyse CT: Nervenkompression Truncus lumboscralis links. j Detailanalyse CT: Quantifizierung der Innenrotationsfehlstellung links. k Zusammenfassung aller Befunde im 3-DCT. I Planung der Operationsschritte mit: 1. Rückenlage, Nervendekompression über modifizierte Lumbotomie links, Osteotomie vorderer Beckenring rechts und hinterer Beckenring links (nahe SI-Gelenk); 2. Bauchlagerung mit Komplettierung der Osteotomie links und Beckenbodendissektion, Reposition und präliminare Stabilisierung; 3. Rückenlage mit Spaninterposition am vordereren Beckenring rechts und Komplettierung der Osteosynthese.

Die beste Prävention vor Beckenfehlstellungen und Pseudarthrosen ist eine frühzeitige Reposition und Notfallstabilisation. Ist diese nicht möglich, sollte Kontakt zu einem spezialisierten Zentrum aufgenommen werden!

In dem vorliegenden Beitrag sollen die von uns im Verlauf der letzten 10 Jahre weiterentwickelten Konzepte zur Indikationsstellung und Operationsdurchführung exemplarisch dargestellt und anhand von Fallbeispielen erläutert werden. Obwohl die Indikationen stetig er-
Azetabulum in zunehmendem Maße rekonstruktive Maßnahmen nach posttraumatischen Früh- und Spätschäden zur Anwendung kommen, sollen an dieser Stelle exemplarisch, ohne Anspruch auf Vollständigkeit, Therapiemöglichkeiten dargestellt werden.

\section{Hauptteil}

\section{Indikationsstellung}

Das Ziel der Behandlung nach instabilen Beckenringverletzungen vom Typ $\mathrm{C}$ ist eine Ausheilung in möglichst exakt anatomischer Stellung. Als kritisch, d.h. mit einer höheren Rate von späteren Beschwerden begleitet, werden posteriore Fehlstellungen von $>5 \mathrm{~mm}$ im hinteren Beckenring und $>10 \mathrm{~mm}$ im vorderen Beckenring angesehen. Die aktuellen Behandlungskonzepte erlauben es, bei weit über $85 \%$ der Patienten eine derartig gute Ausheilungsposition zu erreichen. Indikationen zur Spätkorrektur bestehen, wenn der Patient nachvollziehbare, beckenbedingte Schmerzen hat und eine Fehlstellung von mindestens $2 \mathrm{~cm}$ besteht bzw. eine schmerzhafte Pseudarthrose nachweisbar ist. Da Pseudarthrosen i. d. R. chirurgisch einfacher zu behandeln sind, sollte in diesen Fällen eine operative Korrektur eher früh erfolgen. Ist die knöcherne Heilung eingetreten, kann es günstiger sein, zunächst eine weitere Weichteilkonsolidierung abzuwarten.

Klinisch wichtige Zeichen sind die Beinverkürzung im Stehen (mit und ohne Hüftgelenkskontraktur), die sog. „sitting imbalance" (Tuber ischiadica stehen nicht auf gleicher Höhe, „Umkippen“ beim Sitzen auf hartem Untergrund) und parasakrale Druckstellen und Schmerzen von der Dorsalverschiebung einer Beckenhälfte. Eine exakte Anamnese und klinische Untersuchung ergibt häufig auch Hinweise auf beckenbedingte neurologische und urologische Defizite (Blasen-/Mastdarmstörungen, Fußheber-/-senker-Schwäche/-Parese, Blasenentleerungsstörungen, erektile Dysfunktion, Dyspareunie, externe und interne Fisteln etc.), die weitergehende Diagnostik bedingen. Nicht selten liegen auch posttraumatische Verarbeitungsstörungen vor, die vom Patienten auf eine ggf. unerhebliche Beckenproblematik fokussiert werden und nicht chirurgisch behandelbar sind! Idealerweise folgt man einem umfassenden interdisziplinär aufgestellten Untersuchungsprotokoll. Im eigenen Vorgehen gehen jeder Indikationsstellung mindesten 2 
ambulante Vorstellungstermine in unserer Beckensprechstunde voraus, um eine tragfähige Vertrauensbasis für beide Seiten zu schaffen.

Bevor eine Beckenkorrektur in Erwägung gezogen werden kann, müssen alle Befunde und die individuellen Lebensumstände des Patienten bekannt sein!

Die radiologische Analyse stützt sich auf die komplette Vordiagnostik, insbesondere, wenn Osteosynthesen durchgeführt wurden, um Instabilitätsausmaß, Fehleinschätzungen in der Klassifikation und resultierende sekundäre Dislokationen, überwundene und ggf. noch floride Infekte etc., genau evaluieren zu können. Aktuell werden zunächst eine Beckenübersichtsaufnahme, Inlet- und Outletaufnahmen, ggf. auch Ala- und Obturatoraufnahmen angefertigt. Diese Aufnahmen erlauben schon eine recht genaue quantitative Analyse von Translationsfehlstellungen und der Richtung der häufigen Rotationsfehlstellungen. Eine feinschichtige Spiral-CT-Untersuchung wird zunächst benötigt, um Pseudarthrosen von Fehlheilungen zu unterscheiden. Sie wird mit allen notwendigen multiplanaren Reformationen zusätzlich verwendet, um den ehemaligen Frakturverlauf und das Frakturmuster zu erkennen und ggf. Zusatzpathologien $\mathrm{zu}$ diagnostizieren (Nervenwurzelkompressionen, Lage der Organe wie z.B. Blase, Rektum etc.). Auch das Ausmaß und die Richtung von Rotationsfehlstellungen kann nun gemessen werden.

Bei speziellen Fragestellungen, wie z.B. Läsionen des Plexus lumbosacralis, werden NMR-Untersuchungen angeschlossen, um z.B. den Verlauf der einzelnen Nervenwurzeln zu analysieren.

Bei folgenden Befunden wird im eigenen Vorgehen derzeit eine operative Beckenkorrektur vorgeschlagen:

- Schmerzen, Beschwerden auf die Beckensituation zurückzuführen

- Beinverkürzung und/oder „sitting imbalance“ über $2 \mathrm{~cm}$, Ausgleich durch Schuherhöhung/Kissen führt nicht zur Beschwerdebesserung

- radiologisch bestehende Pseudarthrose oder Fehlheilung

- Weichteildeckung lässt Eingriff zu bzw. plastische Deckung erreichbar

- Patient mental in der Lage, die Eingriffsrisiken $\mathrm{zu}$ erfassen und psychisch in der Lage, einen langfristigen Behandlungsprozess durchzustehen (Heilungszeit 4-6 Monate)

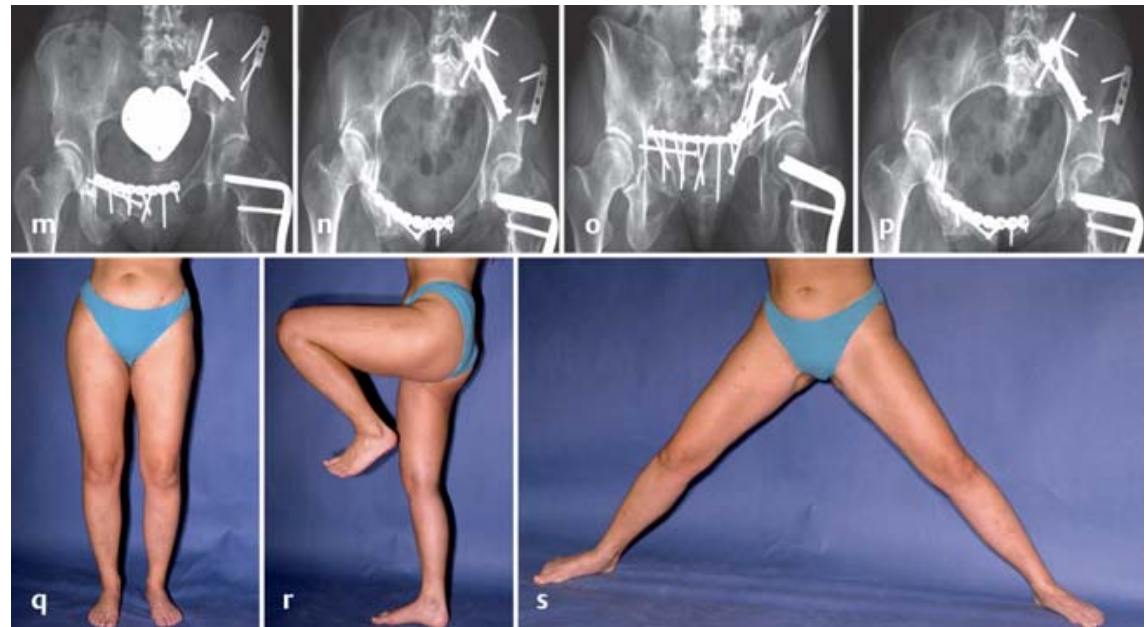

Abb. $\mathbf{1} \mathbf{m}$ bis $\mathbf{s} \mathbf{~ m}$ Postoperatives Ergebnis Beckenübersichtsaufnahme. $\mathbf{n}$ Postoperatives Ergebnis Inletaufnahme. o Postoperatives Ergebnis Outletaufnahme. p Radiologisches Ergebnis (Beckenübersicht) nach 1 Jahr, Patientin rehabilitiert, beschwerdefrei, Nervenschaden komplett erholt. q-s Klinisches Ergebnis nach 1 Jahr.

\section{Operationsplanung (Abb. 1)}

Die Operationsplanung muss folgende Grundgegebenheiten berücksichtigen:

Ziel der Operation ist die möglichst exakte Wiederherstellung der Beckenposition. Trotz kompletter vorderer und hinterer Osteotomie des Beckenrings kann weder eine Translation noch eine ausreichende Rotation der Beckenhälfte erreicht werden, wenn nicht der komplette Beckenboden inklusive der teilweise erheblichen Vernarbungen und der Beckenbodenbänder (Lig. sacrotuberale, Lig. sacrospinale) operativ gelöst wird. Die Durchtrennung muss am sakralen Ursprung erfolgen (Bauchlage), um Verletzungen des $\mathrm{N}$. pudendus zu vermeiden. Repositionen in Bauchlage können allerdings erschwert sein und gelingen nur, wenn der vordere Beckenring zuvor gelöst/osteotomiert wurde. Die Reposition benötigt auch nach kompletter Lösung erhebliche Kräfte, die in der Regel nur mit Spezialinstrumentarium (Fixationseinrichtungen am Tisch und/oder Extensionseinrichtungen) übertragen werden können. Vernarbungen im Bereich des Plexus lumbosacralis und/oder $\mathrm{N}$. ischiadicus können bei Traktion über $1-2 \mathrm{~cm}$ zu sekundären Nervenausfällen führen, Neurolysen müssen daher ggf. primär geplant werden. Korrekturen über $5-7 \mathrm{~cm}$ haben ein sehr hohes Risiko eines iatrogenen Nervenschadens, hier sind ggf. zweizeitige Eingriffe und/oder Dauerextensionsbehandlungen zu erwägen. Die Korrektur von Innenrotationsverletzungen führt zu Knochendefekten im vorderen Beckenring. Es sind ggf.
Knochentransplantationen zur Defektauffüllung zu planen.

Unter Berücksichtigung dieser Grundregeln erfolgt entsprechend der individuellen Voraussetzungen die Festlegung der Osteotomie- und Lagerungssequenz sowie der einzelnen Repositions- und Stabilisierungsschritte. Die Detailplanung kann virtuell am 3-D-Modell erfolgen, bei schwierigen Fällen ist ggf. die Anfertigung eines Hartschaumstoffmodells mit Probeosteotomien hilfreich. Idealerweise erfolgen auch am Becken die Osteotomien in der ehemaligen Fraktur. Transforaminale und zentrale Sakrumfehlstellungen können allerdings nicht intraläsionär osteotomiert werden, in der Regel wird auf transalare Osteotomien ausgewichen. Sekundäreffekte an den Nervenwurzeln müssen berücksichtigt und ggf. gezielte Dekompressionen vorgeschaltet werden. Navigiert gesteuerte Osteotomien erlauben i.d.R. Osteotomien in oder knapp neben der Läsion, erhöhen aber Operationszeit und damit auch den Blutverlust. Eine ggf. mehrtägige intensivmedizinische Betreuung muss sichergestellt sein.

\section{Vorbereitung}

Neben den allgemeinen Operationsvorbereitungen und der Bereitstellung von 6 Erythrozythenkonzentraten werden präoperativ abführende Maßnahmen durchgeführt.

Die Lagerung erfolgt idealerweise auf dem röntgendurchlässigen Operationstisch, der Einsatz von Bildwandler, Ex- 


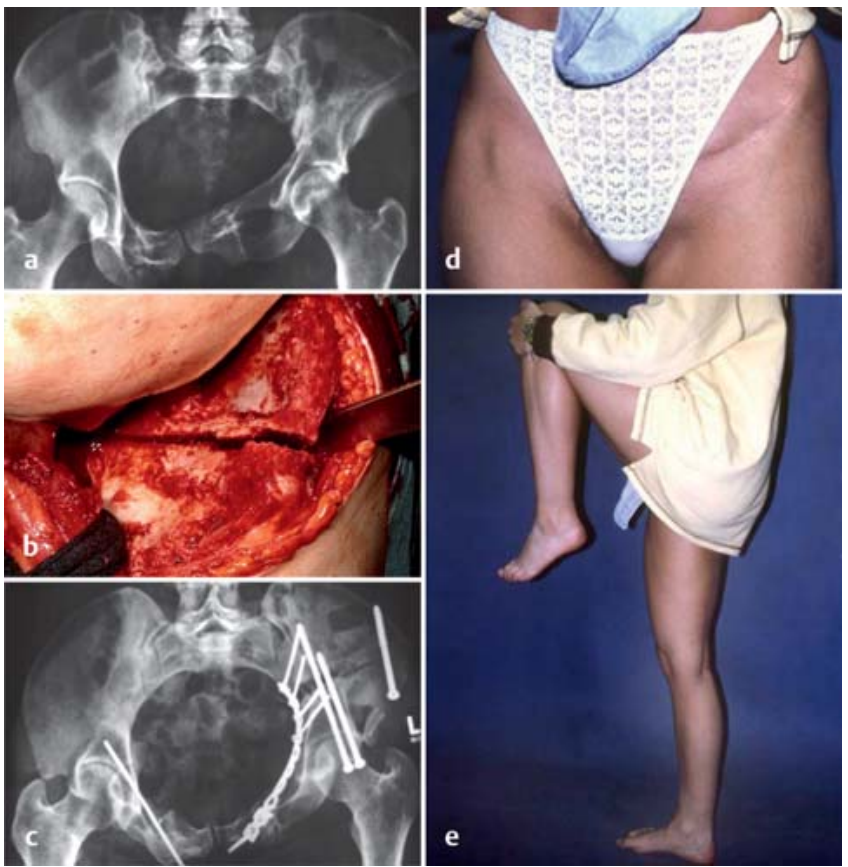

Abb. 2 a bis a 26-jährige Patientin, 1 Jahr nach Beckenfraktur Typ C (transiliakal links und transpubisch beidseits). b Intraoperative Ansicht: transiliakale Osteotomie. c Postoperative Röntgenkontrolle. d Klinisches Bild nach 1 Jahr, Patientin beschwerdefrei, im alten Beruf rehabilitiert. e Klinisches Bild nach 1 Jahr.
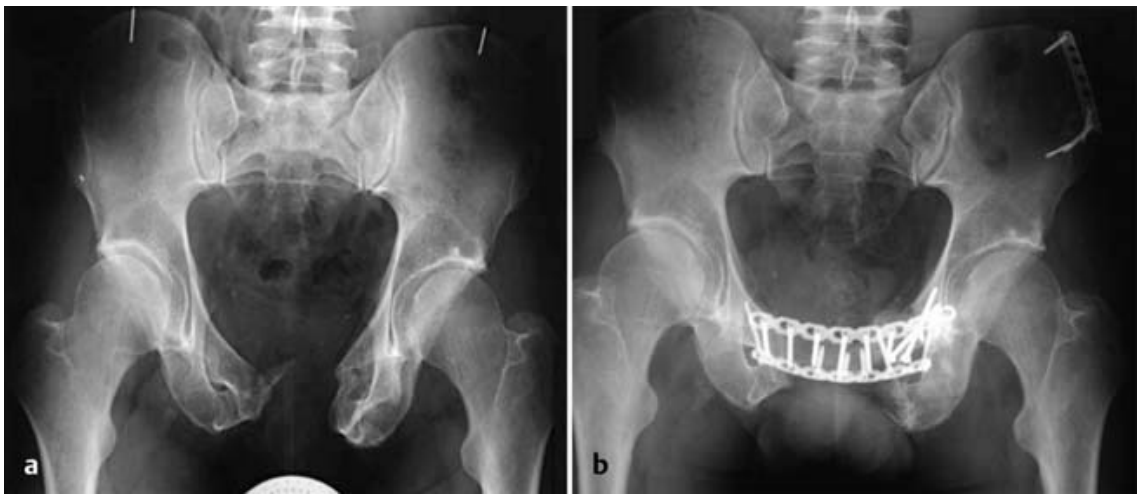

Abb. 3 a und b a 41-jähriger Patient, 11 Jahre nach Motorradunfall mit komplexer (Blasenruptur) vorderer Beckenringverletzung Typ A (transpubische Verletzung bds. und transsymphysär). Stark zunehmende Beschwerden und Hernienbildung. b Zustand nach vorderer Beckenringkorrektur mit beidseitiger oberer und unterer Schambeinastosteotomie und Spaninterposition.

tensionseinrichtung, Fixationseinrichtung mit Tischkopplung etc. wird vor der Abdeckung geprüft. Ggf. wird auch der Einsatz des 3-D-Bildwandlers und/ oder der Navigation vorbereitet. Bildwandler mit großem Flachbilddetektor erleichtern die Kontrolle der Reposition, da beide Beckenhälften gleichzeitig dargestellt werden können. Neuromonitoring wird im eigenen Vorgehen und in Übereinstimmung mit internationalen Erfahrungen nicht eingesetzt. Eine Wärmeeinrichtung sollte eingesetzt werden, da die lange Operationszeit und die großen Wundflächen nahezu zwangsläufig zur Auskühlung und zu Gerinnungsstörungen führen.

\section{Operationsdurchführung (Abb. 2 und 3)}

Der Eingriff wird plangerecht in ständiger Abstimmung mit der Anästhesie durchgeführt. Insbesondere okkulte Blutverluste durch die Osteotomieflächen und ein Auskühlen des Patienten müssen beachtet werden!

\section{Nachbehandlung}

Intensivmedizinische Betreuung, in der Regel Nachbeatmung bis zur ausreichenden Aufwärmung. Nach Extubation sofortige DMS-Kontrolle (Cave: Fehldiagnosen durch Schmerzkatheter!).

Dränagen werden nach 48 Stunden entfernt, eine Frühmobilisation mit Vollbelastung der nicht osteotomierten Seite wird angestrebt. Die osteotomierte Beckenhälfte wird, je nach Situation, für 816 Wochen teilbelastet.

Klinische Kontrollen inkl. einer orientierenden neurologischen Untersuchung (bei Ausfällen auch neurologisches Konsil und elektrophysiologische Untersuchung sowie Verlaufskontrollen!) in der Regel im Raster 6, 12 Wochen, 6, 12, 24 Monate, bei Bedarf auch häufiger.

Röntgenkontrollen: postop. Beckenübersicht, nach Verschraubungen auch mit CT-Untersuchung. Im Bedarfsfall zusätzliche Beckenübersicht nach Mobilisation.

Nach 6 Wochen Beckenübersichtsaufnahme und Inlet-/Outletaufnahmen, nach 12 Wochen Beckenübersicht und CT-Untersuchung (knöcherne Heilung?). Nach 6 Monaten, 1 Jahr und 2 Jahren Beckenübersichtsaufnahmen.

\section{Ergebnisse}

Die eigene Erfahrung umfasst derzeit 41 Patienten, die Indikationen sind in Tab. 1 dargestellt. Bei 30 Patienten konnte eine deutliche Besserung des Ausgangsbefundes, teilweise bis zur kompletten Rehabilitation und Reintegration erreicht werden. Die genaue Aufarbeitung der Ergebnisse ist Gegenstand einer getrennten Untersuchung.

\section{Fehlheilungen nach Azetabulumfrakturen}

Die operative Behandlung nach Azetabulumfrakturen orientiert sich weiterhin an den grundlegenden Untersuchungen und Erfahrungen von E. Letournel. In großen Serien von spezialisierten Chirurgen können in etwa 70-80\% der Fälle gute Langzeitergebnisse erreicht werden, ansonsten werden etwa 50-60\% gute Langzeitergebnisse berichtet. Spätkorrekturen werden selten durchgeführt und betreffen im Wesentlichen unzureichende Repositionen und intraartikuläre Fehlstellungen $[2,5]$.

Zusätzlich problematisch ist, neben dem ungenügenden Repositionsergebnis, der primäre Knorpelschaden, der häufig im Ausmaß nicht quantifizierbar ist und dann zu Früharthrosen führt. Knorpelersatztherapien wurden an Hüftkopf und Azetabulum bisher nur in Einzelfällen durchgeführt. Im eigenen Vorgehen wird seit 2008 unter strenger Indikationsstellung die Knochen-KnorpelTransplantation aus dem Kniegelenk mit Diamanthohlfräsen angewendet, um 


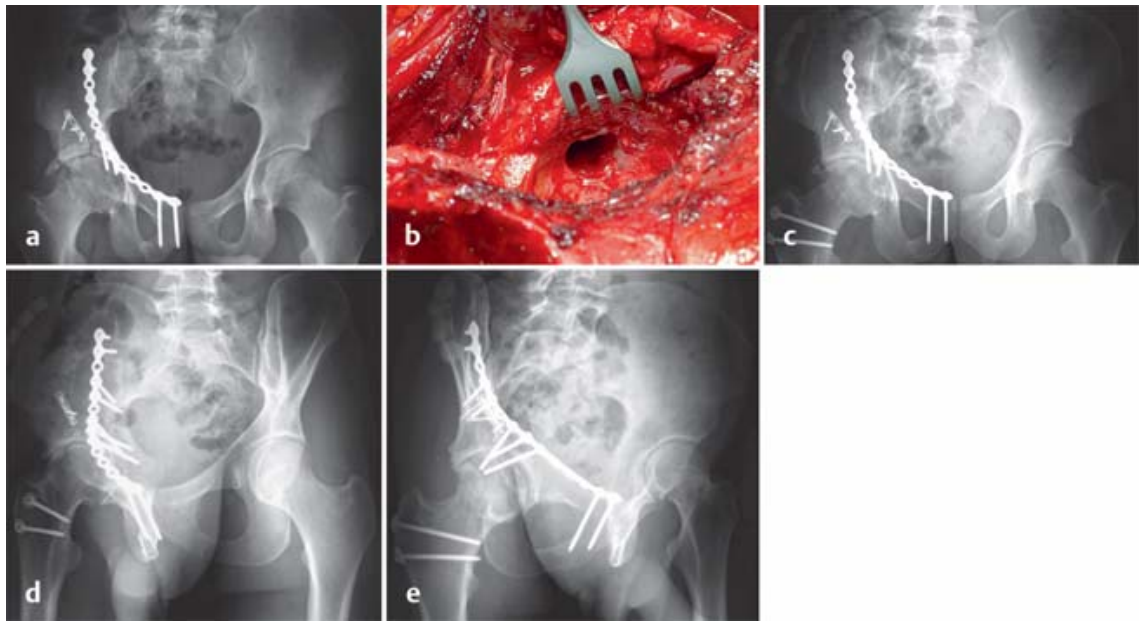

Abb. 4a bis e a 19-jähriger Patient, 18 Monate nach operativer Versorgung einer Azetabulumfraktur (T-Fraktur) über den Ilioinguinalen Zugang mit jetzt zunehmender posttraumatischer Arthrose bei zentralem azetabulärem Knorpeldefekt und korrespondierendem Knorpelschaden am Hüftkopf. b Intraoperativer Befund mit Vorbereitung der Knochen-Knorpel-Transplantation mit der Diamanthohlschleife zur Auffüllung des azetabulären Defekts. Ausgleich des Knorpelschadens am Hüftkopf mit 3 Knorpel-Knochenzylindern, ebenfalls vom gleichseitigen Knie. c Radiologischer Befund nach 18 Monaten. Patient zwischenzeitlich wieder als auszubildender Dachdecker arbeitsfähig. d Radiologischer Befund nach 18 Monaten (Ala-Projektion). e Radiologischer Befund nach 18 Monaten (Obturatorprojektion).

zerstörten Knorpel zu ersetzen (Abb. 4). Die ersten Frühergebnisse sind bisher gut, an der technischen Optimierung der Methode zum Einsatz am Azetabulum wird derzeit gearbeitet.

\section{Schlussfolgerung}

Korrekturosteotomien zur Behandlung posttraumatischer Fehlstellungen und Pseudarthrosen nach instabilen Beckenringfrakturen sind bei korrekter Primärbehandlung nur selten indiziert. Ein standardisiertes Behandlungskonzept frischer Beckenverletzungen sollte daher auch immer den frühen kollegialen Aus- tausch z.B. innerhalb der Traumanetzwerke einschließen, um ggf. die in der günstigen Frühphase bis 3 Wochen nach der Verletzung wesentlich einfachere anatomische Rekonstruktion und Stabilisierung der Beckenverletzung zu ermöglichen. Bei eingetretener Fehlstellung ist eine genaue Analyse notwendig, um eine ggf. vorliegende Indikation zur Spätkorrektur zu erkennen und ein individuelles Behandlungskonzept zu erstellen. Die sich ständig verbessernden operativen Möglichkeiten erlauben heutzutage auch in vielen dieser bisher „aussichtslosen Fälle“ eine deutliche Verbesserung der Lebensqualität und Reintegration.

\section{Literatur}

${ }^{1}$ Gautier E, Rommens PM, Matta JM. Late reconstruction after pelvic ring injuries. Injury 1996; 27 (Suppl. 2): B39-B46

2 Johnson EE, Matta JM, Mast JW et al. Delayed reconstruction of acetabular fractures 21120 days following injury. Clin Orthop Relat Res 1994; 305: 20-30

${ }^{3}$ Kanakaris NK, Angoules AG, Nikolaou VS et al. Treatment and outcomes of pelvic malunions and nonunions: a systematic review. Clin Orthop Relat Res 2009; 467: 2112-2124

${ }^{4}$ Matta JM, Dickson KF, Markovich GD. Surgical treatment of pelvic nonunions and malunions. Clin Orthop Relat Res 1996; 329: 199-206

${ }^{5}$ Mayo KA, Letournel E, Matta JM et al. Surgical revision of malreduced acetabular fractures. Clin Orthop Relat Res 1994; 305: 47-52

${ }^{6}$ Mears DC. [Management of pelvic pseudarthroses and pelvic malunion]. Orthopade 1996; 25: 441-448

7 Oransky $M$, Tortora $M$. Nonunions and malunions after pelvic fractures: why they occur and what can be done? Injury 2007; 38: 489496

8 Taller S, Srám J, Lukás $R$ et al. [Nonunions or malunions of pelvic fractures]. Acta Chir Orthop Traumatol Cech 2009; 76: 121-127

9 Vanderschot P, Daenens K, Broos P. Surgical treatment of post-traumatic pelvic deformities. Injury 1998; 29: 19-22

Prof. Dr. med. Tim Pohlemann Ärztlicher Direktor Priv.-Doz. Dr. med. Jörg Holstein Stationsarzt

Priv.-Doz. Dr. med. Ulf Culemann Ltd. Oberarzt

Klinik für Unfall-, Hand- und Wiederherstellungschirurgie Universitätsklinikum Homburg/Saar Kirrberger Straße 66421 Homburg

tim.pohlemann@ uniklinikum-saarland.de 